\title{
Immune response of cattle immunized with a conjugate of the glycolipid glucose monomycolate and protein
}

\author{
Thi Kim Anh Nguyen ${ }^{a}$, Willemien Wieland ${ }^{a}$, Wiebren Santema ${ }^{\text {ab }}$, Jeroen Hoeboer ${ }^{a}$, \\ Willem van Eden ${ }^{\mathrm{a}}$, Victor Rutten ${ }^{\mathrm{ac}}$, Ad Koets ${ }^{\mathrm{ab}}$, Ildiko Van Rhijn ${ }^{\mathrm{a} *}$
}

* Corresponding author. Tel.: +31 030 2534345; fax: +31 030 2533555. E-mail address: i.vanrhijn@uu.nl (I. Van Rhijn).

\author{
a Department of Infectious Diseases and Immunology, Faculty of Veterinary Medicine, Utrecht University, Yalelaan 1,3584CL, Utrecht, \\ The Netherlands \\ b Department of Farm Animal Health, Faculty of Veterinary Medicine, Utrecht University, Yalelaan 1,3584CL, Utrecht, The \\ Netherlands \\ ${ }^{c}$ Department of Veterinary Tropical Diseases, Faculty of Veterinary Science, University of Pretoria, Private BagX04, Onderstepoort \\ 0100, South Africa
}

\begin{abstract}
Strong anti glycolipid lgG responses can occur in humans and animals, but contrary to anti protein responses and anti glycoprotein responses, the exact mechanism of induction is unknown. We have previously shown that experimental immunization with the glycolipid glucose monomycolate (GMM) causes the development of specific T cell responses, but not of anti GMM antibodies. However, cattle naturally infected with Mycobacterium avium ssp. paratuberculosis produce high levels of anti GMM IgG. In the present study, we tested whether vaccination with GMM conjugated to a protein mimics natural infection in its capacity to induce the production of antibodies against GMM. Cattle were immunized ( $n=5$ per group) with GMM conjugated to a protein, or GMM and protein non-conjugated and administered at contralateral locations, or carrier only. Although immunization with the GMM-protein conjugate vaccine and the non-conjugated vaccine induced protein specific antibody responses, GMM specific antibodies were not detected in either of the groups. In conclusion, the generation of isotype-switched anti lipid antibodies appears to require more than providing peptide epitopes for T helper cells to support glycolipid specific B cells in antibody production.
\end{abstract}

Keywords:

Vaccine

Immunoglobulin

Mycobacterium tuberculosis

Mycobacterium bovis

Glycolipid

Conjugate vaccine

Cattle

1. Introduction

Mycobacterial infections cause enormous health problems world wide. A distinguishing feature of mycobacteria is their thick cell wall, rich in lipids and glycolipids, which form a strong physical barrier. It has been suggested that anti lipid antibodies may contribute to protection against mycobacterial infection (Lang and Glatman-Freedman, 2006).

Antibody responses to lipoarabinomannan are thought to be relevant in the protection against Mycobacterium tuberculosis in humans and mice (Costello et al., 1992; Fischer et al., 2004; Hamasur et al., 2003).

Similar to proteins, lipid antigens have the capacity to elicit $T$ cell proliferative responses in a number of species including humans (Moody et al., 2000), mice (Kinjo et al, 2006), guinea pigs (Hiromatsu et al., 2002), and cattle (Nguyen et al., 2009; Van Rhijn et al., 2009). We have recently described the cell-mediated and humoral immune responses in cattle upon vaccination with the glycolipid glucose monomycolate (GMM), an abundant component of the mycobacterial cell wall (Nguyen et al., 2009). A vaccine containing GMM as the only antigen elicited T cell responses but no antibody responses while a vaccine with a pure protein as the only antigen generated both $T$ cell and antibody responses (Nguyen et al, 2009). In general, the generation of antibodies of the IgG isotype requires T cell help. It has recently been shown in mice that presentation of a-galactosylceramide and related compounds by CD1d on B cells can attract strong T cell help (Barral et al., 2008; Leadbetter et al., 2008; Galli et al., 2003). However, in humans and cattle, GMM is presented by CD1b, which is not expressed by B cells (Van Rhijn et al, 2006; Girardi et al., 2010). In addition, it has been suggested that GMM-specific T cells do not home to the lymph nodes, where B cell maturation takes place (Nguyen et al., 2009). However, M. avium ssp. paratuberculosis infected cattle are known to mount an antibody response to GMM (Nguyen et al., 2009) which could possibly be caused by fragments of bacterial cell walls that function as a conjugate vaccine because they contain cell wall lipids and proteins. We therefore hypothesized that a conjugate of GMM with a protein may provide T cell help for B cells to produce antibodies against surface exposed glycolipids on mycobacteria, analogous to the glycoconjugate vaccines that have successfully been designed to induce antibodies against polysaccharides. 
The aim of the current study was to investigate if GMM specific antibodies are generated when GMM is coupled to protein and used in an experimental vaccine. Keyhole limpet haemocyanin (KLH) is an immunogenic model antigen in cattle as we have shown previously (Nguyen et al., 2009). As an alternative protein component of the experimental conjugate vaccine we used the ubiquitous $70 \mathrm{kD}$ heat shock protein (Hsp70) of $M$. avium ssp. paratuberculosis, the bacterium that causes a strong GMM antibody responses during infection. Hsp70 is known to induce a(3 T cell responses and humoral responses (Koets et al., 1999, 2001; Bonorino et al, 1998). We immunized cattle with GMM$\mathrm{KLH}$ or GMM-Hsp70 conjugate vaccines, or non-conjugated vaccines of which the GMM and protein components were delivered in contralateral shoulders, or vaccine without immunogenic protein or GMM, and evaluated both cell mediated and antibody mediated immune responses.

\section{Materials and methods}

\subsection{Conjugate vaccines}

\subsubsection{Antigens}

M. phlei was cultured in $7 \mathrm{H} 9$ medium (Difco) supplemented with $1 \%$ glucose and $0.05 \%$ Tween-80 (Sigma-Aldrich). Bacteria were washed in distilled water, spun down and extracted in chloroform:methanol 1:2 and 2:1 consecutively for 2 hat room temperature. This total lipid extract was fractionated on a LC-Silica Packing column (Supelco) and GMM was prepared as described (Nguyen et al, 2009). KLH (Sigma-Aldrich) and Hsp70 (production described previously by Koets et al., 2001) were biotinylated using D-biotinoyl- $\varepsilon$-aminocaproic acid N-hydroxysuccinimide-ester (Roche) in DMSO according to the supplied protocol. Free biotin was removed from biotinylated proteins by EconoPac 10DG column.

\subsubsection{Vaccine preparation}

The

(DOPC)

and

lipids

1,2-dipalmitoyl-sn-glycero-3- 1,2-dioleoyl-sn-glycero-3-phosphocholine

phosphoethanolamine-N-(cap biotinyl) (PE-biotin) from Avanti Polar Lipids were used to prepare liposomes (as described in Barral et al., 2008). Mixtures of DOPC, PE-biotin, and GMM (88:2:10, m:m:m) or DOPC and PEbiotin(98:2, m:m) were prepared in glass tubes and dried under a stream of nitrogen, followed by high speed vacuum to remove all traces of solvent. Liposomes were made by cryofracture of the dried lipid mixtures inTris- $\mathrm{HCl} 25 \mathrm{mM}$, $\mathrm{NaCl} 50 \mathrm{mM}, \mathrm{pH}$ 8. Liposomes were incubated with 150 (jLg of 50mg/ml silica microspheres per vaccine dose (100 $\mathrm{nm}$; Kisker GbR), washed once in PBS and once in blocking buffer (PBS, 1\% BSA, 1\% FCS), followed by incubation with $1 \mathrm{Jtg} / \mathrm{ml}$ streptavidin (Sigma) for $20 \mathrm{~min}$ at room temperature and another wash with blocking buffer. At this point, the production of "GMM only" vaccines (microspheres with GMM containing liposomes) and the "carrier only" vaccines (microspheres with liposomes that lack GMM) was completed. For the KLH and Hsp70-containing vaccines, a subsequent incubation with 300 ljig biotinylated protein was performed, followed by two washes in FACS buffer. All vaccines were resuspended in $1.5 \mathrm{ml}$ PBS for injection. The contents of the vaccines are summarized in Table 1.

To demonstrate the presence of the lipid species, aliquots of vaccines were loaded on a TLC plate and resolved in chloroform:methanol:water 60:16:1.5 (v:v:v), dried, and sprayed with 3\% cupric acetate in 8\% phosphoric acid, dried, and baked at $150^{\circ} \mathrm{C}$ for about $1 \mathrm{~h}$ to visualize GMM, DOPC, and PE. The presence of the proteins in the vaccines was assessed by dot blot and Western blot analyses. Aliquots of Hsp70-containing vaccines were boiled in Laemmli loading buffer and separated on a $14 \%$ polyacrylamide gel, along with prestained protein ladder (Fermentas), and transferred to nitrocellulose membrane.

Recombinant Hsp70 was detected using mouse anti Hsp70 (clone KoKo.B02) (Santema et al, 2010) goat anti mouse IgG AP (Southern Biotech) and NBT/BCIP (Roche). KLH is too large to be efficiently transferred to a nitrocellulose membrane by blotting, so $\mathrm{KLH}$-containing vaccines and a $\mathrm{KLH}$ standard of know concentration were denatured by boiling with laemmli buffer, spotted directly to the nitrocellulose membrane, blocked, and incubated with polyclonal rabbit anti $\mathrm{KLH}$ (Gene Tex), followed by mouse anti rabbit IgG AP (Sigma) and NBT/BCIP (Roche). 
Table 1

Vaccine components and treatment of groups.

\begin{tabular}{|c|c|}
\hline Vaccine & Components \\
\hline GMM-KLH conjugate & GMM/KLH/microspheres/DOPC/PE ${ }^{\text {biotin }} /$ streptavidin \\
\hline GMM-Hsp70 conjugate & GMM/Hsp70/microspheres/DOPC/PE ${ }^{\text {biotin }} /$ streptavidin \\
\hline GMM only & $\mathrm{GMM} / \mathrm{microspheres/DOPC} / \mathrm{PE}^{\text {biotin }} /$ streptavidin (no protein) \\
\hline KLH only & $\mathrm{KLH} /$ microspheres/DOPC/PE ${ }^{\text {biotin } / \text { streptavidin (no GMM) }}$ \\
\hline Hsp70 only & Hsp70/microspheres/DOPC/PE biotin/streptavidin (no GMM) \\
\hline Canier only & Microspheres/DOPC/PE biotin/streptavidin (no GMM, no protein) \\
\hline Group ( $n=5$ per group) & Vaccines given (shoulder) \\
\hline GMM-KLH conjugate & GMM-KLH conjugate (left); carrier only (right) \\
\hline GMM-Hsp70 conjugate & GMM-Hsp70 conjuage (left); carrier only (right) \\
\hline GMM-KLH non-conjugated & GMM only (left); KLH only (right) \\
\hline GMM-Hsp70 non-conjugated & GMM only (left); Hsp70 only (right) \\
\hline Carrier only & Carrier only (left); carrier only (right) \\
\hline
\end{tabular}

\subsection{Animals and immunization}

Twenty five Holstein-Friesian calves ( 3 weeks of age) were purchased and housed for two weeks before the first immunization, which was considered to be experimental day 0 . Blood was drawn from the jugular vein in heparinized and uncoated Vacutainer tubes (BD) 7 days before the first immunization, two blood samplings, at day 14 and 28 , were performed between the first and second immunization, which took place at day 35 , and three samplings took place after the second immunization, on day 49,70 , and after 75 days. To investigate the stimulation of humoral and cellular responses of calves to lipid-protein conjugates, 5 animals were immunized subcutaneously with GMM-KLH conjugate vaccine and 5 animals were immunized with GMM-Hsp70 conjugate vaccines. There were two nonconjugated vaccine control groups: one group received a GMM only vaccine in one shoulder and KLH only vaccine in the contralateral shoulder, and another group received GMM only vaccine in one shoulder and Hsp70 only vaccine in the contralateral shoulder. One additional control group received the carrier only in both shoulders. The exact contents of the vaccines given to each of the groups are listed in Table 1. Sera of animals suffering from clinical paratuberculosis and of animals that were vaccinated with inactivated whole cell mycobacteria (Gudair, CZ Veterinaria, Spain) were included in this study. Paratuberculosis caused by natural exposure to $M$. avium ssp. paratuberculosis was diagnosed by a fecal culture at the National Veterinary Health Service (GD Deventer, The Netherlands). Experiments were approved by the Animal Ethical Committee of the University of Utrecht, The Netherlands.

\subsection{Enzyme-linked immunosorbent assays}

To detect antigen-specific immunoglobulins, Polysorb plates (Nunc, Denmark) were coated with GMM (5 $\mu \mathrm{g} / \mathrm{well}$, dissolved in methanol, and dried overnight in a fume hood), and Costar high binding 96-well plates were used to coat proteins $\left(0.1 \mathrm{jjig} /\right.$ well, dissolved in PBS, and coated overnight at $\left.4^{\circ} \mathrm{C}\right)$. All plates were blocked for $1 \mathrm{~h}$ with blocking buffer (Roche), before serum was added in threefold dilutions, starting at 1:30, and plates were incubated overnight at $4{ }^{\circ} \mathrm{C}$. Plates were washed 3 times with PBS- $0.25 \%$ Tween- 20 or PBS- $0.5 \%$ Tween- 20 respectively before adding biotinylated mouse anti bovine total IgG (Sigma-Aldrich) diluted 1:50,000 in blocking buffer for $1 \mathrm{~h}$, followed by 3 washes, incubation with by Avidin PO (BD Pharmigen) 1:1000 in blocking buffer, and 3 washes with PBS without Tween. ABTS (Roche) was used as PO substrate and the ELISA plates were read by spectrophotometer at $405 \mathrm{~nm}$.

\section{4. $T$ cell proliferation assays}

PBMC were isolated using Histopaque-1077 (Sigma-Aldrich) ficoll centrifugation. Lipid and protein antigens were added to 96-well round bottom plates in series of dilutions, starting at $15 \mu \mathrm{g} / \mathrm{ml}$. GMM was dried in stream of nitrogen to remove organic solvent, and sonicated in RPMl 1640 (Gibco) with $25 \mathrm{mM} \mathrm{HEPES}$ supplemented with 10\% FCS (Hyclone), penicillin and streptomycin (Gibco), and $2 \mathrm{mM}$ glutamax (Gibco). PBMC $\left(2 \times 10^{5} /\right.$ well) were added to the plates and incubated in a $5 \% \mathrm{CO} 2$ incubator at $37^{\circ} \mathrm{C}$ for 3 days. Proliferation was measured by a $7 \mathrm{~h}$ pulse of $1 \mu \mathrm{Ci}$ of $\left[{ }^{3} \mathrm{H}\right]$ thymidine before cells were harvested and $(\beta$-emission counted. Stimulation indices represent the counts/min of triplicate wells stimulated with antigen divided by the counts/min of triplicate wells stimulated with medium without antigen. 
A

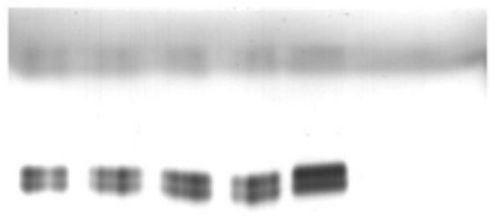

$\frac{7}{2}+\frac{7}{4} \cdot \frac{}{6} \cdot \frac{}{7}$
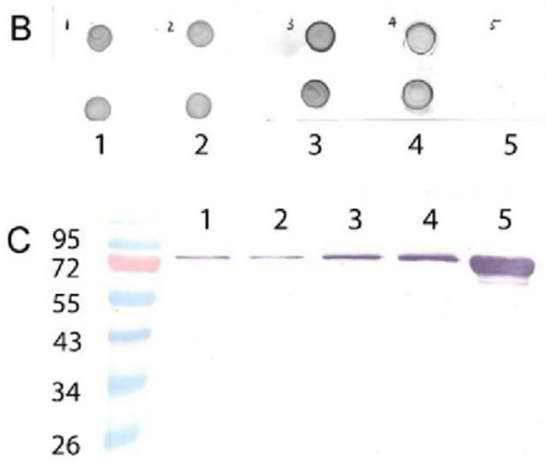

Fig. 1. Protein and lipid content of vaccines. Upon finalization of the conjugated and non-conjugated vaccines, the amount of GMM in the vaccines was analyzed by TLC (A). Of each GMM-containing vaccine, $25 \%$ of a vaccine dose was loaded in lane 1-4. Lane 1: GMM-KLH conjugate vaccine; lane 2: GMM-Hsp 70 conjugate vaccine; lane 3-4: GMM only vaccine; lane 5: pure GMM standard (50 $\mu$ ); lane 6: DOPC (25 $\mu \mathrm{g}$ ); lane 7: PE (25 $\mu \mathrm{g}$ ). KLH in KLHcontaining vaccines was analyzed by dot blot (B). KLH was detected by polyclonal rabbit anti KLH antibody. In 1 and 2 , each duplicate dot contains $6 \%$ of a vaccine dose. 1: GMM-KLH conjugate vaccine; 2 : KLH only vaccine; 3 : biotinylated KLH (6 $\mu$ g); 4 : non-biotinylated KLH (6 $\mu$ g); 5 : Hsp70 as a negative control $(6 \mu \mathrm{g})$. Hsp70 was detected in Hsp 70-containing vaccines by Western blotting, using mouse anti Hsp 70 antibody for detection (C). Lane 1: $3 \%$ of a GMM-Hsp 70 conjugate vaccine; lane 2: $3 \%$ of a Hsp70 only vaccine; lane 3: $6 \%$ of a GMM-Hsp70 conjugate vaccine; lane $4: 6 \%$ of a Hsp70 only vaccine; lane 5: $6 \mu \mathrm{g}$ biotinylated Hsp70.

\subsection{Statistics}

ANOVA and two-tailed Student's t-test from GraphPad Prism 4.0 and Microsoft Office Excel 2003 were used. Differences between groups are considered significant if $P<0.05$.

\section{Results and discussion}

\subsection{Protein can be efficiently conjugated to GMM in a vaccine}

To be able to investigate the effect of GMM conjugation to protein on the anti GMM antibody response, we used a method of non covalent association of protein and lipid to microspheres (Barral et al., 2008). For a valid comparison between the non-conjugated and conjugate vaccines, it is crucial that the amount of GMM is equal in both types of vaccine, as well as the amount of protein. The amounts of the other vaccine components like the carrier lipids DOPC and $P E$, and the microspheres were standardized for all types of vaccines. The quantity of GMM in conjugate and non-conjugated vaccines was analyzed by TLC (Fig. 1A) and showed a comparable amount of this antigen in both types of vaccines, which was estimated to be 100 (jig per dose. The quantity of KLH and Hsp70 was analyzed by dot blot and Western blot respectively (Fig. 1B and C). Equal amounts of each protein were present in conjugate and non-conjugated vaccines, which were estimated to be 20 ( $\mu \mathrm{g}$ per dose. Of note: all vaccines, including carrier only, were prepared with carrier lipids, streptavidin, and microspheres as described in Section 2 and summarized in Table 1.

\subsection{GMM-protein conjugate vaccine does not induce \\ anti GMM antibodies}

One group of animals ( $n=5$ in all groups) was immunized with GMM-KLH conjugate vaccine in one shoulder and carrier only in the contralateral shoulder; one group was injected with GMM only vaccine in one shoulder and KLH only vaccine in the contralateral shoulder and one group of animals received carrier only in both shoulders. Total serum IgG antibody against GMM and KLH was examined by ELISA (Fig. 2). As compared to the control vaccination, carrier only, GMM specific antibodies were not detected in either of the two groups after immunization (Fig. 2B, D and F). Some animals showed a weak IgG response against GMM before the first immunization, which disappeared several weeks later (Fig. 2F), which can be explained by the presence of maternal antibodies. As expected, a strong IgG antibody response against KLH was seen in animals immunized with KLH separately (Fig. 2E) as compared to carrier only immunized animals $(P<0.05)$. Although weaker responses were detected in 2 out of 5 animals that were immunized with GMM-KLH conjugate vaccines, the anti KLH antibody response of this whole group was also significantly higher than the carrier only immunized group $(P<0.05)$ (Fig. 2A).

In addition, we also included two groups immunized with GMM-Hsp70 conjugate and non-conjugated vaccines. A similar pattern was seen when we used Hsp70 instead of KLH (Supplemental Fig. 1A, B, D and E). 
In the group of animals that received non-conjugated GMM and Hsp70 vaccines, one animal showed an anti GMM IgG response (Supplemental Fig. 1F). In addition, one animal in the group that was injected with carrier only showed a strong anti Hsp70 IgG response from day 7 till day 14 of the experiment, which dropped at 49 days after the first immunization (Supplemental Fig. 1C).

From these experiments it becomes clear that vaccination with a GMM-protein conjugate does not recapitulate the anti GMM antibody inducing capacity of a natural infection. When tested in parallel with the sera of the GMM-KLH or the GMM-Hsp70 conjugate vaccine immunized animals, a markedly higher IgG reactivity against GMM was seen in animals that were naturally infected with $M$. avium ssp. paratuberculosis $(P<0.05)$. To investigate whether infection with a live bacterium is necessary to induce GMM antibodies we also tested sera of animals that were vaccinated subcutaneously with the Gudair vaccine, which consists of inactivated (non-replicating) $M$. avium ssp. paratuberculosis. Though lower than the responses of animals infected with live $M$. avium ssp. paratuberculosis, Gudair vaccinated animals did show a significant anti GMM IgG response compared to the carrier vaccinated group $(P<0.05)$ (Fig. 2G). From this we conclude that additional mycobacterial components rather than factors associated with active infection with the pathogen can stimulate the immune system to induce anti GMM antibody response.

\subsection{Immunization induces weak $T$ cell responses against GMM}

In addition to the evaluation of the antibody responses, we examined T cell activity against GMM and proteins in animals that were immunized with GMM-protein conjugate vaccine, non-conjugated vaccine and carrier only. $T$ cell proliferation assays were carried out before immunization and after immunization until 1.5 months after the second immunization using freshly isolated PBMC. A higher, but non significant response against GMM was detected in animals that were immunized with GMM-containing conjugate or non-conjugated vaccines compared to carrier only vaccinated animals (Fig. $3 \mathrm{~A}$ and $\mathrm{C}$ ). Lack of significance of $\mathrm{T}$ cell responses could be due to the limited number of blood samplings after the second immunization as compared to our previously published GMM vaccination studies (Nguyen et al., 2009).

\subsection{Non-conjugated vaccine induces stronger anti protein $T$ cell and antibody responses than conjugate vaccine}

Because the amount of protein in the conjugate and non-conjugated vaccines was equal, we expected that the anti protein antibody responses and the anti protein $T$ cell responses would be comparable. However, as noted in Section 1 on antibody responses, all animals that received $\mathrm{KLH}$ in a non-conjugated vaccine responded with a strong anti KLH IgG responses, but only three out of five animals that received GMM-KLH conjugate vaccine showed a comparably strong response (Fig. 2A and E). The difference in anti KLH lgG response between the conjugate and non conjugate vaccine immunized groups was however not statistically significant. Also in animals immunized with non-conjugated vaccine containing Hsp70, higher IgG response to Hsp70 were detected than in animals receiving the GMM-Hsp70 conjugate, and this case the difference between the groups was significant $(P<0.05)(S u p p l e m e n t a l$ Fig. $1 \mathrm{~A}$ and $\mathrm{E}$ ).

The differences between conjugate and non-conjugated vaccines in the generation of anti protein antibody responses were mirrored by a difference in anti protein $\mathrm{T}$ cell responses. Even though the total amount of antigens administered was equal, the difference in anti KLH T cell responses between non-conjugated KLH and conjugate GMM-KLH groups was significant $(\mathrm{P}<0.05)$ (Fig. 3B). No significant differences were detected in $\mathrm{T}$ cell proliferation of animals that were immunized with Hsp70 in non-conjugated or conjugate vaccines compared to animals vaccinated with carrier only (Fig. 3D).

Our data show that conjugation of GMM to a protein down regulates or prevents an optimal antibody response against that protein. One possible explanation is that GMM masks or changes epitopes of the protein. Why antibodies against GMM itself are not generated upon vaccination is not clear. During natural infection with $\mathrm{M}$. avium ssp. paratuberculosis or vaccination with inactivated bacteria (the Gudair vaccine), proteins and GMM are also "conjugated" in the bacterial cell wall, yet strong anti GMM antibody responses develop. Additional stimulatory components present in mycobacteria, like TLR agonists, may be responsible for the difference between conjugate vaccine and exposure to bacterial cell walls. Inclusion of these compounds in a GMM-protein conjugate vaccine may improve the impaired $T$ cell responses that were observed using the conjugate vaccine, and this could possibly overcome the lack of development of anti GMM IgG antibodies (Pasare and Medzhitov, 2005; Banchereau and Steinman, 1998). 
Anti KLH lgG response
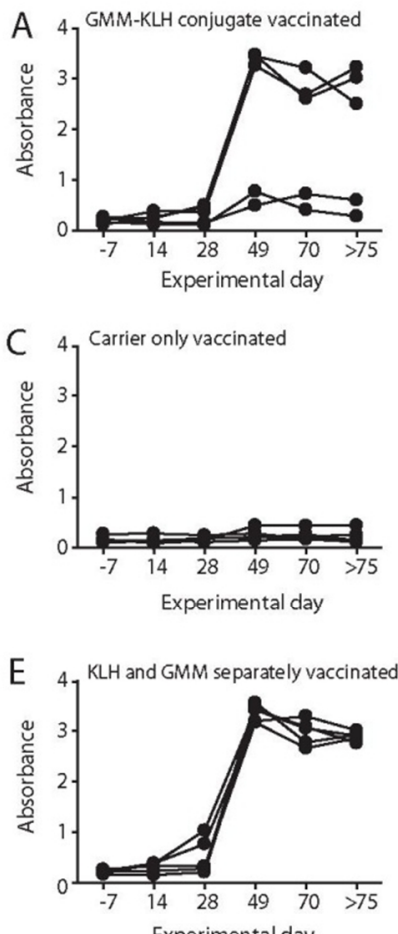

Anti GMM lgG response
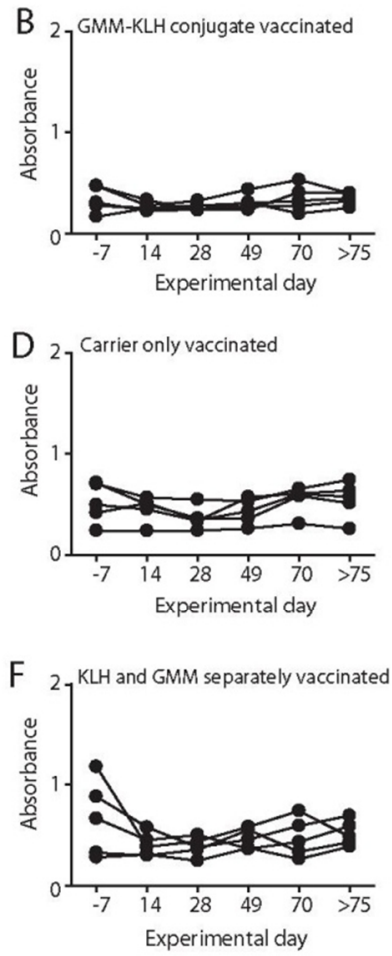

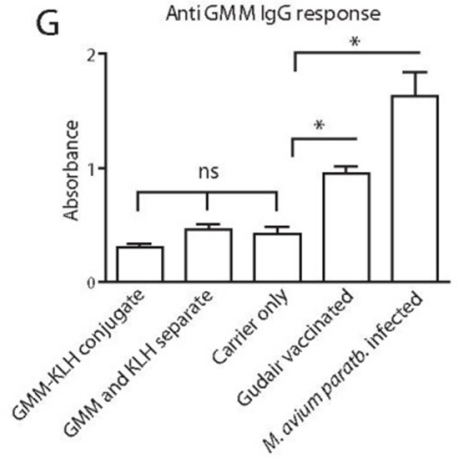

Fig. 2. Antibody responses against lipid and protein vaccine components. (A-F) Total IgG responses against KLH (left panels) and $M$. phlei GMM (right panels) of conjugate GMM-KLH vaccinated animals (A and B); carrier vaccinated animals ( $C$ and $D$ ); and non-conjugated GMM and KLH vaccinated animals ( $\mathrm{E}$ and $\mathrm{F}$ ) was determined by ELISA. Animals received the first immunization on day 0 and the second one on day 35 of the experiment. Symbols connected with a line represent data from one individual animal. (G) GMM specific IgG in animals two weeks after the second immunization with the three experimental vaccines, three weeks after the second immunization with the Gudair vaccine, and in animals chronically infected for 2-4 years with $M$. avium ssp. paratuberculosis. Each bar represents data from 5 animals. Statistically significant differences $(P \leq 0.05)$ are marked with *. 

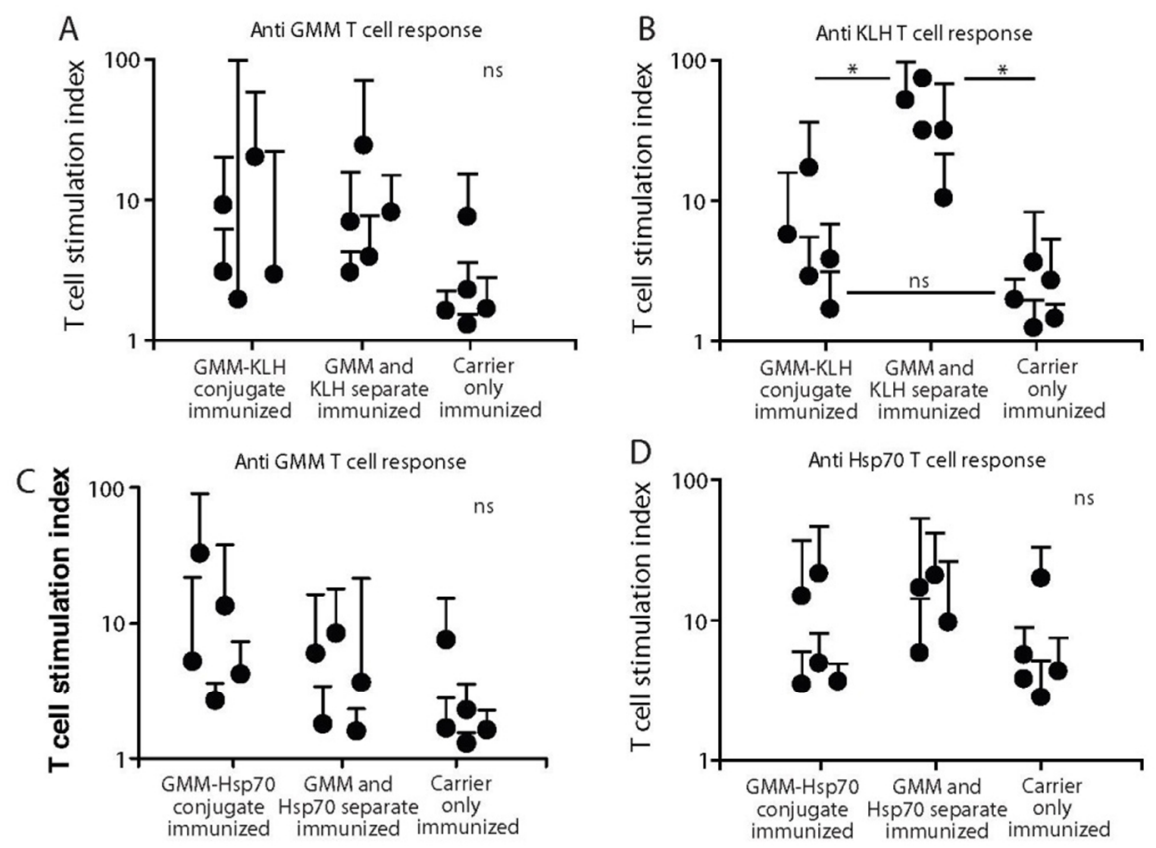

Fig. 3. T cell responses against lipid and protein vaccine components. (A-D) PBMC freshly isolated from blood were stimulated for three days with $M$. phiei GMM (A and C), KLH (B), and Hsp70 (D). Each symbol represents the average value of 5 independent experiments carried out on one animal post immunization. The T cell stimulation index was calculated by dividing the counts/min of the antigen stimulated wells divided by counts/min of medium (without antigen) after a 7-h pulse of [3H] thymidine. Statistically significant differences $(P \leq 0.05)$ are marked with *.

\section{Acknowledgements}

I. Van Rhijn is supported by a NWO Meervoud subsidy (\#836.08.001), and T.K.A. Nguyen is supported by the Ministry of Education and Training of Vietnam according to Decision 322/QD-TTg for Overseas Training Projects.

\section{Appendix A. Supplementary data}

Supplementary data associated with this article can be found as a separated attachment.

\section{References}

Banchereau, J., Steinman, R.M., 1998. Dendritic cells and the control of immunity. Nature 392, 245-252.

Barral, P., Eckl-Dorna, J., Harwood, N.E., De Santo, C, Salio, M., Illarionov, P., Besra, G.S., Cerundolo, V., Batista, F.D., 2008. B cell receptor-mediated uptake of CD1d-restricted antigen augments antibody responses by recruiting invariant NKT cell help in vivo. Proc. Natl. Acad. Sci. U.S.A. 105 (24), 8345-8350.

Bonorino, C, Nardi, N.B., Zhang, X., Wysock, L.J., 1998. Characteristics of the strong antibody response to mycobacterial Hsp70: a primary T celldependent IgG response with no evidence of natural priming or gdTcell involvement. J. Immunol. 161,5210-5216.

Costello, A.M., Kumar, A., Narayan, V., Akbar, M.S., Ahmed, S., Abou-Zeid, C, Rook, G.A., Stanford, J., Moreno, C, 1992. Does antibody to mycobacterial antigens including lipoarabinomannan, limit dissemination in childhood tuberculosis? Trans. R. Soc. Trop. Med. Hyg. 86, 686-692.

Fischer, K., Scotet, E., Niemeyer, M., Koebernick, H., Zerrahn, J., Maillet, S., Hurwitz, R., Kursar, M., Bonneville, M., Kaufmann, S.H.E., Schaible, U.E., 2004. Mycobacterial phosphatidylinositol mannoside is a natural antigen forCD1d-restrictedT cells. Proc. Natt. Acad. Sci. U.S.A. 101 (29), 10685-10690.

Galli, G., Nuti, S., Tavarini, S., Galli-Stampino, L, De Lalla, C, Casorati, G., Dellabona, P., Abrignani, S., 2003. CD1d-restricted help to B cells by human invariant natural killer T lymphocytes. J. Exp. Med. 197 (8), 1051-1057.

Girardi, E., Wang, J., Mac, T.T., Versluis, C, Bhowruth, V., Besra, G., Heck, J.R.A., Van Rhijn, I., Zajonc, M.D., 2010. Crystal structure of bovine CD1b3 with endogenously bound ligands.J. Immunol. 185 (1), 376-386.

Hamasur, B., Haile, M., Pawlowski, A., Schröder, U., Williams, A., Hatch, G., Hall, G., Marsh, P., Källenius, G., Svenson, S.B., 2003. Mycobacterium tuberculosis arabinomannan-protein conjugates protect against tuberculosis. Vaccine 21,4081-4093.

Hiromatsu, K., Dascher, C.C., LeClair, K.P., Sugita, M., Furlong, S.T., Brenner, M.B., Porcelli, S.A., 2002. Induction of CD1-restricted immune responses in guinea pigs by immunization with mycobacterial lipid antigens. J. Immunol. 169,330-339.

Kinjo, Y., Tupin, E., Wu, D., Fujio, M., Garcia-Navarro, R., Mohammed Rafii-El-Idrissi Benhnia, M.R.E.I., Zajonc, D.M., Ben-Menachem, G., Ainge, G.D Painter, G.F., Khurana, A., Hoebe, K., Behar, S.M., Beutler, B., AWil-son, I., Tsuji, M., Sellati, T.J., Wong, C.H., Kronenberg, M., 2006. Natural killer T cells recognize diacylglycerol antigens from pathogenic bacteria. Nat. Immunol. 7 (9), 978-986.

Koets, A.P., Rutten, V.P., de Boer, M., Bakker, D., Valentin-Weigand, P., van Eden, W., 2001. Differential changes in heat shock protein-, lipoarabinomannan-, and purified protein derivative-specific immunoglobulin G1 and G2 isotype responses during bovine Mycobacterium avium subsp.paratuberculosis infection. Infect. Immun. 69 (3), 1492-1498.

Koets, A.P., Rutten, V.P., Hoek, A., Bakker, D., van Zijderveld, F., Müller, K.E., van Eden, W., 1999. Heat-shock protein-specific T-cell responses in 
various stages of bovine paratuberculosis. Vet. Immunol. Immunopathol. 70 (1-2), 105-115.

Lang, M.L., Glatman-Freedman, A., 2006. Do CD1-restricted T cells contribute to antibody-mediated immunity against Mycobacterium tuberculosis? Infect. Immun. 74 (2), 803-809.

Leadbetter, E.A., Brigl, M., Illarionov, P., Cohen, N., Luteran, M.C., Pillai, S., Besra, G.S., Brenner, M.B., 2008. NK T cells provide lipid antigen-specific cognate help for B cells. Proc. Natl. Acad. Sci. U.S.A. 105 (24), 8339-8344.

Moody, D. B., T. Ulrichs, W. Muhlecker, D. C. Young, S. S. Gurcha, E. Grant, J. P. Rosat, M. B. Brenner, C. E. Costello, G. S. Besra and S. A. Porcelli, 2000. "CD1c-mediated T-cell recognition of isoprenoid glycolipids in Mycobacterium tuberculosis infection." Nature. 20 404(6780), 884-888.

Nguyen, T.K.A., Koets, A.P., Santema, W.J., Van Eden, W., Rutten, V.P.M.G., Van Rhijn, I., 2009. The mycobacterial glycolipid glucose monomyco-late induces a memory $\mathrm{T}$ cell response comparable to a model protein antigen and no $\mathrm{B}$ cell response upon experimental vaccination of cattle. Vaccine $27,4818-4825$.

Pasare, C, Medzhitov, R., 2005. Control of B-cell responses by Toll-like receptors. Nature 438, 364-368.

Santema, W., van Kooten, P., Hoek, A., Leeflang, M., Overdijk, M., Rutten, V., Koets, A., 2010. Hsp70 vaccination-induced antibodies recognize B cell epitopes in the cell wall of Mycobacterium avium subspecies paratuberculosis. Vaccine 29 (7), 1364-1373.

Van Rhijn, I., Koets, A.P., Im,J.S., Piebes, D., Reddington, F., Besra, G.S., Porcelli, S.A., Van Eden, W., Rutten, V.P.M.G., 2006. The Bovine CD1 Family Contains Group 1 CD1 Proteins, but no functional CD1d. J. Immunol. 176, 4888-4893.

Van Rhijn, I., Nguyen, T.K.A., Michel, A., Cooper, D., Govaerts, M., Cheng, T.Y., Van Eden, W., Moody, D.B., Coetzer, A.W.J., Rutten, V., Koets, A.P., 2009. Low cross-reactivity of T-cell responses against lipids from Mycobacterium bovis and M. avium paratuberculosis during natural infection. Eur.J. Immunol. 39 (11), 3031-3041. 